\title{
High-Quality AlGaN/GaN Grown on Sapphire by Gas-Source Molecular Beam Epitaxy using a Thin Low-Temperature AIN Layer
}

\section{J. Jurkovic, L.K. Li, B. Turk, W. I. Wang}

Department of Electrical Engineering, Columbia University, New York, NY, 10027

\section{S. Syed, D. Simonian, H. L. Stormer}

Department of Physics, Columbia University, New York, NY, 10027

\section{ABSTRACT}

Growth of high-quality $\mathrm{AlGaN} / \mathrm{GaN}$ heterostructures on sapphire by ammonia gassource molecular beam epitaxy is reported. Incorporation of a thin AlN layer grown at low temperature within the GaN buffer is shown to result in enhanced electrical and structural characteristics for subsequently grown heterostructures. AlGaN/GaN structures exhibiting reduced background doping and enhanced Hall mobilities (2100, 10310 and $12200 \mathrm{~cm}^{2} / \mathrm{Vs}$ with carrier sheet densities of $6.1 \times 10^{12} \mathrm{~cm}^{-2}, 6.0 \times 10^{12} \mathrm{~cm}^{-2}$, and $5.8 \mathrm{x}$ $10^{12} \mathrm{~cm}^{-2}$ at $300 \mathrm{~K}, 77 \mathrm{~K}$, and $0.3 \mathrm{~K}$, respectively) correlate with dislocation filtering in the thin AlN layer. Magnetotransport measurements at $0.3 \mathrm{~K}$ reveal well-resolved Shubnikov-de Haas oscillations starting at $3 \mathrm{~T}$.

\section{INTRODUCTION}

A unique combination of electrical, thermal, chemical, and structural characteristics render the $\mathrm{AlGaN} / \mathrm{GaN}$ material system extremely suitable for application in high-speed, high-power electronic applications. Attractive properties include: a wide bandgap (3.4 for GaN, $6.2 \mathrm{eV}$ for AlN), large breakdown field ( 3 MV/cm), high peak and saturation electron velocities $\left(\sim 3 \times 10^{7} \mathrm{~cm} / \mathrm{s}\right)$, robust structural and thermal stability, and chemical inertness. The large conduction band offset at the AlGaN/GaN interface and large spontaneous and piezoelectric polarization fields in the strained heterostructure enable the formation of a high-density two-dimensional electron gas (2DEG) on the order of $10^{13} \mathrm{~cm}^{-2}$ in AlGaN/GaN heterostructures grown in the (0001) direction. The Ga- or Aface is uniquely identified by reflection high-electron energy diffraction (RHEED) patterns exhibiting $2 \times 2,5 \times 5$, or $6 \times 4$ surface reconstructions as opposed to the $\mathrm{N}$ - or $\mathrm{N}$ face which exhibit $1 \times 1,3 \times 3,6 \times 6$ and $c(6 \times 12)$ patterns [1]. Thus, the same role fullfilled by sheet-doping in modulation-doped FET's (MODFET's) is achieved in the AlGaN/GaN system without the mobility-limiting scattering mechanisms present in intentionallydoped structures. Consequently, piezoelectrically-doped high electron mobility transistor (HEMT) structures exhibiting high-speed, high-power operation and which can be subject to adverse environmental conditions can be achieved utilizing the $\mathrm{AlGaN} / \mathrm{GaN}$ system.

AlGaN/GaN HEMT's which exhibit promising high-frequency, high-temperature, and high-power characteristics have been achieved [2-5] in spite of the lack of a latticematched substrate. Enhanced device characteristics for HEMT's grown on $6 \mathrm{H}-\mathrm{SiC}$ substrates have been partially attributed to the higher thermal conductivity and closer lattice match to $\mathrm{GaN}$ (3.4\%) as compared to that for sapphire (13.8\%). AlGaN/GaN 
heterostructures exhibiting mobilities of $2000 \mathrm{~cm}^{2} / \mathrm{Vs}, 9000 \mathrm{~cm}^{2} / \mathrm{Vs}$, and $11000 \mathrm{~cm}^{2} / \mathrm{Vs}$ with sheet densities of $1 \times 10^{13} \mathrm{~cm}^{-2}, 1 \times 10^{13} \mathrm{~cm}^{-2}, 7 \times 10^{12} \mathrm{~cm}^{-2}$ at $300 \mathrm{~K}, 77 \mathrm{~K}$, and $4.2 \mathrm{~K}$, respectively, have been grown on conducting $6 \mathrm{H}-\mathrm{SiC}$ substrates by metalorganic chemical vapor deposition (MOCVD) [6]. The highest attained mobility for similar structures grown on sapphire by MOCVD is $10300 \mathrm{~cm}^{2} / \mathrm{Vs}$ with an electron sheet density of $6.2 \times 10^{12} \mathrm{~cm}^{-2}$ at $1.5 \mathrm{~K}$ in spite of the large lattice mismatch [7]. Recently, latticematched homoepitaxy has been demonstrated by the use of a GaN template grown on sapphire by MOCVD for a plasma-assisted molecular beam epitaxy (PA-MBE) growth process, resulting in mobilities as high as $1150 \mathrm{~cm}^{2} / \mathrm{Vs}, 24000 \mathrm{~cm}^{2} / \mathrm{Vs}$, and $51700 \mathrm{~cm}^{2} / \mathrm{Vs}$ with sheet electron densities of $1.4 \times 10^{13} \mathrm{~cm}^{-2}, 2.5 \times 10^{12} \mathrm{~cm}^{-2}, 2.2 \times 10^{12} \mathrm{~cm}^{-2}$, at 300 $\mathrm{K}, 77 \mathrm{~K}$, and $13 \mathrm{~K}$, respectively, although the template layer was shown to exhibit a high background doping $[8,9]$.

In contrast, $\mathrm{AlGaN} / \mathrm{GaN}$ heterostructures grown directly on highly mismatched (0001)-oriented sapphire substrates have also been achieved by molecular beam epitaxy (MBE) $[10,11]$, resulting in much lower mobilities: $1211 \mathrm{~cm}^{2} / \mathrm{Vs}$, and $5660 \mathrm{~cm}^{2} / \mathrm{Vs}$ with electron sheet densities of $4.9 \times 10^{12} \mathrm{~cm}^{-2}$ and $5 \times 10^{12} \mathrm{~cm}^{-2}$ at $300 \mathrm{~K}$ and $77 \mathrm{~K}$, respectively. However, for organometallic vapor phase epitaxial (OMVPE) growth of $\mathrm{GaN}$ on sapphire, the insertion of one or more low-temperature-grown (LT) -AlN or LT$\mathrm{GaN}$ interlayers within the high-temperature-grown $\mathrm{GaN}$ has recently been shown to result in a reduction in threading dislocation density for subsequently grown epilayers [12]. More specifically, a defect filtering process was observed as a large portion of the stress-induced threading dislocations originating from the GaN/sapphire interface were terminated at the LT-AIN and LT-GaN interlayers, resulting in improved quality for the epilayers grown on the buffer structure. Although experimental data indicates that higher quality growth can be achieved on $6 \mathrm{H}-\mathrm{SiC}$, it remains highly desirable to obtain highquality growth of GaN-based heterostructures on sapphire due to substrate availability, low cost, and high resistivity.

In this work, we report on the incorporation of a single LT-AlN interlayer within the GaN buffer of a AlGaN/GaN HEMT structure grown directly on sapphire (0001) by ammonia gas source MBE (GS-MBE), resulting in a significant enhancement in the structural and electrical characteristics of the subsequently grown heterostructure. AlGaN/GaN structures exhibiting reduced background doping and Hall mobilities of 2100,10310 and $12200 \mathrm{~cm}^{2} /$ Vs with carrier sheet densities of $6.1 \times 10^{12}, 6.0 \times 10^{12}$, and $5.8 \times 10^{12} \mathrm{~cm}^{-2}$ at $300 \mathrm{~K}, 77 \mathrm{~K}$, and $0.3 \mathrm{~K}$, respectively, confirm the effectiveness of the buffer layer structure. The existence of a high-density two-dimensional electron gas is verified by magetotransport measurements performed at $0.3 \mathrm{~K}$ exhibiting Shubnikov-de Haas oscillations for fields as low as $3 \mathrm{~T}$ and a negatively-sloped magnetoresistance, indicating a low background doping in the buffer structure. Finally, a mobility of 2210 $\mathrm{cm}^{2} / \mathrm{Vs}\left(10360 \mathrm{~cm}^{2} / \mathrm{Vs}\right)$ with a sheet charge density $5.5 \times 10^{12} \mathrm{~cm}^{-2}\left(5.9 \times 10^{12} \mathrm{~cm}^{-2}\right)$ for the two-dimensional electron gas at room temperature $(77 \mathrm{~K})$ is calculated based on a two-layer conduction model. The results demonstrate that high-quality AlGaN/GaN HEMT structures can be grown by GS-MBE directly on sapphire in a single growth process by the incorporation of a single LT-AlN interlayer within the GaN buffer.

\section{EXPERIMENT}

AlGaN/GaN HEMT samples were grown on 2"'-diameter, basal-plane sapphire substrates by GS-MBE in a Varian GEN II MBE system equipped with an RF plasma 
source (SVT Associates). Conventional Knudsen effusion cells were used as the Ga and $\mathrm{Al}$ sources while high-purity ammonia gas was used as the nitrogen source. After degreasing, the sapphire substrates were loaded into the MBE system and outgassed at $900{ }^{\circ} \mathrm{C}$ for 30 minutes. All epitaxial layers were unintentionally doped and, with the exception of the AlN buffer layers, grown at $810{ }^{\circ} \mathrm{C}$.

The heteroepitaxial growth was achieving utilizing a two-step growth process [1316]. First, nitridation of the surface was performed at a substrate temperature of $620^{\circ} \mathrm{C}$, followed by low-temperature growth $\left(450^{\circ} \mathrm{C}\right)$ of a thin $(20-30 \mathrm{~nm})$ AlN nucleation layer and an anneal at $810^{\circ} \mathrm{C}$. Second, growth proceeded with the epitaxy of a $3 \mu \mathrm{m}$ undoped$\mathrm{GaN}$ buffer. Growth of the $\mathrm{GaN}$ buffer at $810^{\circ} \mathrm{C}$ was interrupted after $600-700 \mathrm{~nm}$ in order to insert a thin, low-temperature $\left(450{ }^{\circ} \mathrm{C}\right) \mathrm{AlN}$ layer. The thickness of the AlN layer was varied from $20 \mathrm{~nm}$ to $60 \mathrm{~nm}$ for a set of samples for which an optimum thickness of $30 \mathrm{~nm}$ was determined from subsequent van der Pauw Hall characterization.

A $30 \mathrm{~nm}$ AlGaN barrier followed growth of the $\mathrm{GaN}$ buffer. The Al composition for the barriers of the samples reported was determined by x-ray diffraction (XRD) measurements to be close to $20 \%$. In addition, reference samples, consisting of the buffer layer structure both with and without the low-temperature AIN insertion layer, were grown in order to enable estimation of the carrier concentration and mobility of the 2DEG utilizing a two-layer conduction model.

Transport characterization was performed upon prepared samples at room temperature and at $77 \mathrm{~K}$ by van der Pauw Hall measurement. The existence of the piezoelectrically-induced 2DEG at the AlGaN/GaN interface was confirmed by wellresolved Shubnikov-de Haas oscillations observed at $3 \mathrm{~T}$ and above during magnetotransport measurements performed at $0.3 \mathrm{~K}$. Also, RHEED in situ monitoring and atomic force microscopy analysis were employed in order to characterize the structural properties of the epitaxial films while photoluminescence (PL) measurements were performed at room temperature and at $10 \mathrm{~K}$.

\section{RESULTS AND DISCUSSION}

A marked difference in PL spectra for AlGaN/GaN HEMT structures grown with and without the incorporation of the LT-AlN layer within the GaN buffer structure is shown in Figure 1. The spectra were obtained at $10 \mathrm{~K}$. The dominant emission peak, located at $3.490 \mathrm{eV}$, for HEMT samples which incorporate the LT-AIN layer is attributed to transitions associated with free $\mathrm{B}$ excitons $\left(\mathrm{FE}_{\mathrm{B}}\right)$ [17] while that for the samples grown without the AlN layer is ascribed to neutral-bound-excitons with a transition energy of $3.476 \mathrm{eV}$. Also, emission peaks at $3.494 \mathrm{eV}, 3.503 \mathrm{eV}$, and $3.512 \mathrm{eV}$, (the latter two of which are associated with the band-to-band transitions to A and B valence bands, respectively [18]) are readily apparent in the LT-AlN samples while the non-LT-AlN samples exhibited less defined peaks at $3.480 \mathrm{eV}$ and $3.499 \mathrm{eV}$. The highly-resolved A and $\mathrm{B}$ valence band peaks exhibited by the LT-AIN samples correspond with high-quality epitaxial growth, in contrast to the non-LT-AIN samples.

The RHEED patterns for all samples were observed to be relatively streaky. However, enhanced sharpness in the RHEED patterns became apparent during epitaxial growth of the GaN buffer and subsequent layers immediately upon growth of the LT-AIN layer. More specifically, the minor spots and facets observed prior to the LT-AlN layer vanished. In addition, analysis of the samples by atomic force microscopy revealed that the average grain-size for $\mathrm{AlGaN} / \mathrm{GaN}$ heterostructures grown on conventional $\mathrm{GaN}$ 


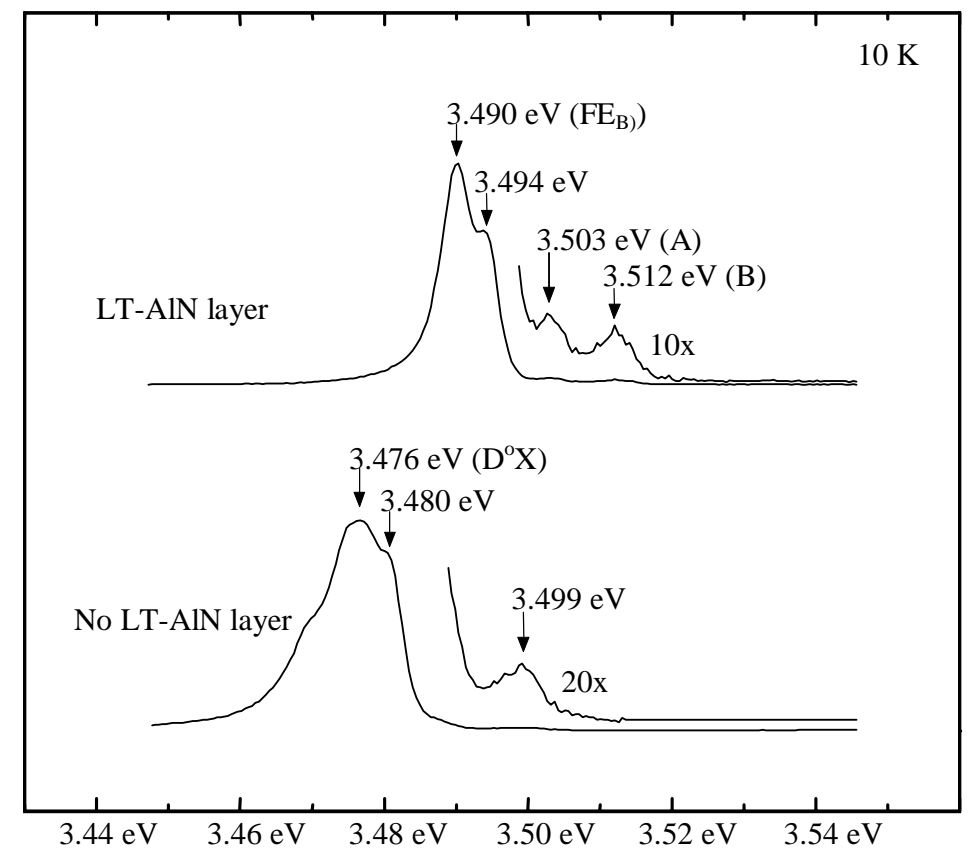

PL Intensity (Arb. Units) vs. Photon Energy

Figure 1. Band edge region of the photoluminescence spectra for $\mathrm{Al}_{0.2} \mathrm{Ga}_{0.8} \mathrm{~N} / \mathrm{GaN}$ samples grown with and without a single LT-AIN interlayer within the GaN buffer.

buffers and those grown on buffers which incorporate the LT-AlN layer, were 200-500 $\mathrm{nm}$ and 1.2-1.5 $\mu \mathrm{m}$, respectively. The line width (FWHM) of x-ray diffraction rocking curve for the heterostructure grown with the LT-AIN interlayer was 100 arc-sec.

The above results obtained from structural and optical characterization indicate a significant enhancement in the crystallinity of the epitaxial layers with the incorporation of the LT-AIN layer and correspond well with the reduced etch pit and threading dislocation density, and superior x-ray diffraction and PL linewidths reported for GaN films grown by OMVPE incorporating single and multiple LT-AIN and LT-GaN interlayers [12]. The enhanced resolution of the PL peaks, increased sharpness of the RHEED patterns, and reduced density of grain boundaries observed in the present work correlate with enhanced electrical characteristics, as confirmed by van der Pauw and magnetoresistance measurements.

The presence of a $2 \mathrm{DEG}$ at the strained $\mathrm{AlGaN} / \mathrm{GaN}$ interface was confirmed by strong and well-resolved Shubnikov-de Haas $(\mathrm{SdH})$ oscillations observed in the magnetoresistance $\left(\mathrm{R}_{\mathrm{XX}}\right)$ measurements at $0.3 \mathrm{~K}$ shown in Figure 2 . The low onset of oscillation, $3 \mathrm{~T}$, indicates a relatively small amount of disorder in the structures while the decreasing minima with increasing magnetic field correlates with minor sub-channel conduction due to a low background doping in the buffer. The electron mobility at $0.3 \mathrm{~K}$ was found to be $12200 \mathrm{~cm}^{2} /$ Vs while an electron sheet density of $5.8 \times 10^{12} \mathrm{~cm}^{-2}$ was determined from the $\mathrm{SdH}$ characteristic. Also, distortion of the $\mathrm{SdH}$ characteristic was 


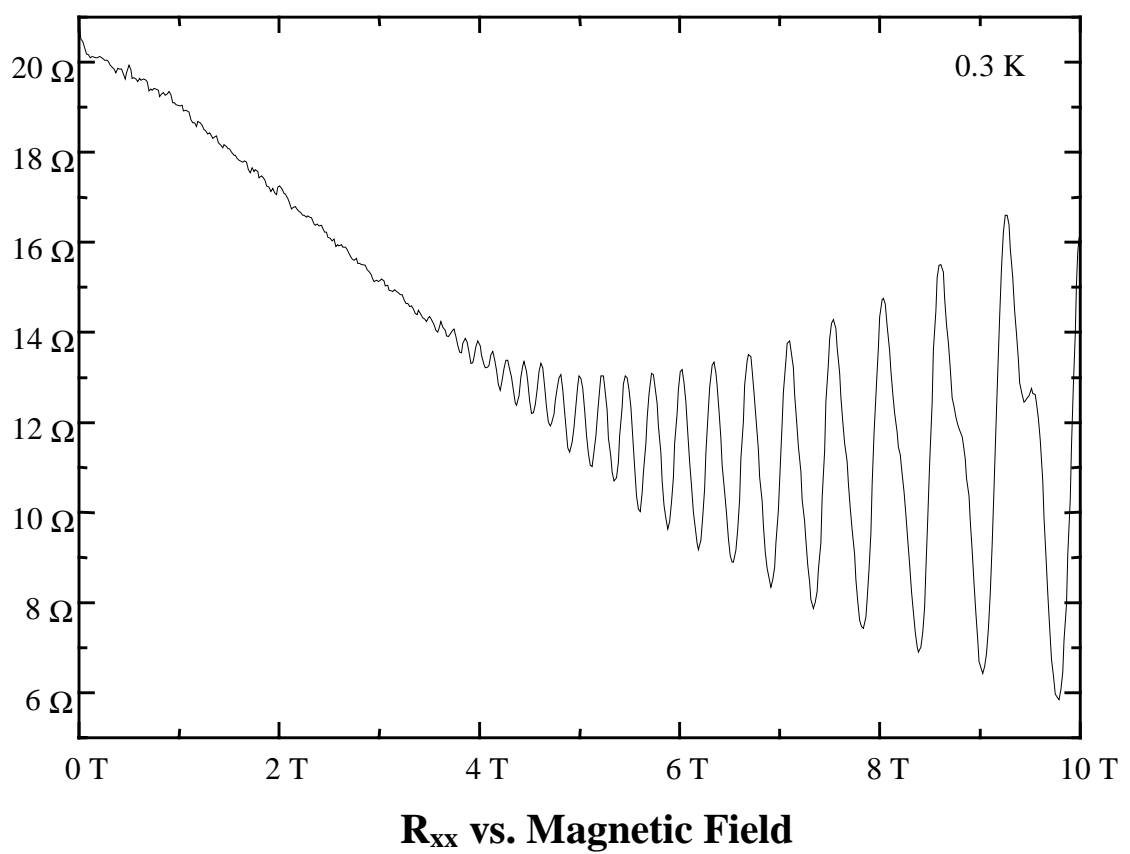

Figure 2. Magnetoresistance, $\mathrm{R}_{\mathrm{Xx}}$, vs. magnetic field at $0.3 \mathrm{~K}$ for an $\mathrm{AlGaN} / \mathrm{GaN}$ heterostructure grown on sapphire by GS-MBE using a buffer which incorporates a LT-ALN interlayer. Shubnikov-de Haas oscillation starts at $3 \mathrm{~T}$.

observed above $7 \mathrm{~T}$ in the form of the appearance of secondary minima near the $\mathrm{SdH}$ maxima. These features may be attributed to spin-splitting of the Landau levels at higher magnetic fields, a phenomenon that is thought to be observable only in crystals of exceptional quality. These additional $\mathrm{SdH}$ features at high magnetic fields have also been recently reported for $\mathrm{AlGaN} / \mathrm{GaN}$ and $\mathrm{GaN}$ structures exhibiting high mobilities achieved by other means $[7,8]$.

The electron mobilities for $\mathrm{AlGaN} / \mathrm{GaN}$ heterostructures grown on $\mathrm{GaN}$ buffers incorporating a single LT-AIN interlayer were determined by van der Pauw Hall measurements to be 2100 and $10310 \mathrm{~cm}^{2} / \mathrm{Vs}$ with carrier sheet densities of $6.1 \times 10^{12} \mathrm{~cm}^{-}$ ${ }^{2}, 6.0 \times 10^{12} \mathrm{~cm}^{-2}$, at $300 \mathrm{~K}$ and $77 \mathrm{~K}$, respectively. A reference buffer sample incorporating the LT-AIN interlayer was found to exhibit a reduced background electron density of $4.7 \times 10^{16} \mathrm{~cm}^{-3}\left(4.3 \times 10^{16} \mathrm{~cm}^{-3}\right)$ at $300 \mathrm{~K}(77 \mathrm{~K})$ as compared to the density, $1.1 \times 10^{17} \mathrm{~cm}^{-3}\left(5.7 \times 10^{16} \mathrm{~cm}^{-3}\right)$, of a buffer sample grown without the LT-AlN interlayer. A mobility of $2210 \mathrm{~cm}^{2} / \mathrm{Vs}\left(10360 \mathrm{~cm}^{2} / \mathrm{Vs}\right)$ with a sheet charge density of 5.5 $\times 10^{12} \mathrm{~cm}^{-2}\left(5.9 \times 10^{12} \mathrm{~cm}^{-2}\right)$ for the two-dimensional electron gas at room temperature $(77 \mathrm{~K})$ is calculated based on a two-layer conduction model using the values of electron sheet density and mobility for the reference GaN buffer sample grown with the LT-AlN interlayer and those obtained above by van de Pauw Hall measurement for the $\mathrm{AlGaN} / \mathrm{GaN}$ heterostructure. The room temperature mobility value for the $2 \mathrm{DEG}$ is the highest among the methods reported for epitaxial growth of $\mathrm{AlGaN} / \mathrm{GaN}$ heterostructures, including plasma-assisted-MBE on MOCVD-grown GaN/sapphire templates, as well as MOCVD or OMVPE on sapphire, 4H-SiC and 6H-SiC [6-11]. 


\section{CONCLUSION}

In conclusion, high quality AlGaN/GaN HEMT structures have been grown directly on sapphire (0001) substrates by GS-MBE in a single growth process. Incorporation of a single LT-AIN interlayer within the GaN buffer is shown to result in significant enhancement in the structural, optical, and electrical transport characteristics of such heterostructures. More specifically, measured electron mobilities were 2100, 10310 , and $12200 \mathrm{~cm}^{2} / \mathrm{Vs}$ with carrier sheet densities of $6.1 \times 10^{12}, 6.0 \times 10^{12}$, and $5.8 \mathrm{x}$ $10^{12} \mathrm{~cm}^{-2}$ at $300 \mathrm{~K}, 77 \mathrm{~K}$, and $0.3 \mathrm{~K}$, respectively. In addition, mobility of the 2DEG was calculated using a two-layer conduction model to be $2210 \mathrm{~cm}^{2} / \mathrm{Vs}\left(10360 \mathrm{~cm}^{2} / \mathrm{Vs}\right)$ with a sheet charge density $5.5 \times 10^{12} \mathrm{~cm}^{-2}\left(5.9 \times 10^{12} \mathrm{~cm}^{-2}\right)$ at room temperature $(77 \mathrm{~K})$. The presence of the $2 \mathrm{DEG}$ was confirmed by well-resolved $\mathrm{SdH}$ oscillations starting at $3 \mathrm{~T}$. A reduction in background doping was observed upon incorporation of the LT-ALN interlayer within the GaN buffer while PL measurements and AFM analysis confirmed a significant decrease in grain boundary and point defect densities. The results demonstrate that a high quality $\mathrm{AlGaN} / \mathrm{GaN}$ heterostructure can be achieved in a single growth step by GS-MBE directly on a sapphire substrate.

\section{ACKNOWLEDGEMENTS}

This work was supported in part by ONR, monitored by Dr. C. E. C. Wood.

\section{REFERENCES}

1. A. R. Smith, R. M. Feenstra, D. W. Greve, M.-S. Shin, M. Skowronski, J. Neugebauer, J. E. Northrup, Appl. Phys. Lett., 72, 2114 (1998).

2. M. A. Khan, Q. Chen, M. S. Shur, B. T. Dermott, J. A. Higgins, J. Burm, W. Schaff, L. F. Eastman, Electron. Lett., 32, 357, (1996).

3. Y.-F. Wu, B. P. Keller, P. Fini, S. Keller, T. J. Jenkins, L. T. Kehias, S. P. Denbaars, U. K. Mishra, Electron Dev. Lett., 19, 50 (1998).

4. R. Li, S. J. Cai, L. Wong, Y. Chen, K. L. Wang, R. P. Smith, S. C. Martin, K. S. Boutros, J. M. Redwing, Electron Dev. Lett., 20, 323 (1999).

5. S. T. Sheppard, K. Doverspike, W. L. Pribble, S. T. Allen, J. W. Palmour, L. T. Kehias, T. J. Jenkins, Electron Dev. Lett., 20, 161 (1999).

6. R. Gaska, M.S. Shur, A. D. Bykhovski, A. O. Orlov, G. L. Snider, Appl. Phys. Lett, 74, 287 (1999).

7. T. Wang, Y. Ohno, M. Lachab, D. Nakagawa, T. Shirahama, S. Sakai, H. Ohno, Appl. Phys. Lett., 74, 3531 (1999). 
8. C. R. Elsass, I. P. Smorchkova, B. Heying, E. Haus, P. Fini, K. Maranowski, J. P. Ibbeston, S. Keller, P. M. Petroff, S. P. Denbaars, U. K. Mishra, J. S. Speck, Appl. Phys. Lett., 74, 3528 (1999).

8. I. P. Smorchkova, C. R. Elsass, J. P. Ibbeston, R. Vetury, B. Heying, P. Fini, E. Haus, S. P. Denbaars, J. S. Speck, U. K. Mishra, J. Appl. Phys., 86, 4520 (1999).

10. L. K. Li, J. Alperin, W. I. Wang, D. C. Look, D. C. Reynolds, J. Vac. Sci. Technol. B, 16, 1275 (1998).

11. J. B. Webb, H. Tang, S. Rolfe, J. A. Bardwell, Appl. Phys. Lett., 75, 953 (1999).

12. H. Amano, M. Iwaya, T. Kashima, M. Katsuragawa, I. Akasaki, J. Han, S. Hearne, J. A. Floro, E. Chason, J. Figiel, Jpn. Journ. Appl. Phys., Part 2, 37, L1540 (1998).

13. W. I. Wang, Appl. Phys. Lett., 44, 1149 (1984).

14. J. S. Harris, S. M. Koch, S. J. Rosner, Mater. Res. Soc. Symp. Proc., 91, 3 (1987).

15. H. Kroemer, J. Crystal Growth, 81, 193 (1987).

16. H. Amano, N. Sawaki, I. Akasaki, Y. Toyoda, Appl. Phys. Lett., 48, 353 (1986).

17. D. C. Renolds, D. C. Look, J. Appl. Phys., 80, 594 (1996).

18. K. C. Zeng, J. Y. Lin, H. X. Jiang, W. Yang, Appl. Phys. Lett., 74, 3821 (1999). 